\title{
Parâmetros ecocardiográficos em modo unidimensional de cães da raça Poodle miniatura, clinicamente sadios ${ }^{1}$
}

\author{
Echocardiographic parameters in unidimensional mode from \\ clinically normal miniature Poodle dogs.
}

\author{
Ronaldo Jun Yamato ${ }^{1}$ Maria Helena Matiko Akao Larsson ${ }^{2}$ \\ Regina Mieko Sakata Mirandola ${ }^{2}$ Guilherme Gonçalves Pereira ${ }^{2}$ \\ Fernanda Lie Yamaki ${ }^{2}$ Ana Carolina Brandão de Campos Fonseca Pinto ${ }^{3}$ \\ Elina Célia Nakandakari ${ }^{4}$
}

\section{RESUMO}

No Brasil, a população canina da raça Poodle, principalmente a variação miniatura, cresce em progressão geométrica, sendo esta raça freqüentemente acometida por cardiopatias congênitas e adquiridas. O escopo deste estudo foi padronizar e avaliar os parâmetros ecocardiográficos em modo unidimensional $(M)$ de cães da raça Poodle miniatura, devido ao aumento populacional da mesma, a variação existente destes parâmetros entre as raças caninas e as diversas cardiopatias às quais os Poodles são predispostos. Foram utilizados 30 cães, da referida raça, sendo 09 machos e 21 fêmeas com idades entre 2 a 7 anos $(3,87 \pm 1,55)$ e peso corpóreo variando de 2,0 a 8,7 quilos $(4,49 \pm 1,38)$. Os cães incluídos neste estudo foram considerados sadios, após terem sido submetidos aos exames físico, laboratoriais, eletrocardiográfico, radiográfico e à mensuração da pressão arterial. Após a realização do exame ecocardiográfico e a análise dos resultados, foi possível obter os valores de referência do exame ecocardiográfico, em modo $M$, para os cães da raça Poodle miniatura e, ainda, sugerir que o peso corpóreo e altura podem exercer influência sobre os parâmetros ecocardiográficos.

Palavras-chave: ecocardiografia, modo $M$, Poodle miniatura, cães.

\section{ABSTRACT}

In Brazil, the canine population of the Poodle, mainly the Miniature variation, grows in geometric progression, beeing this breed frequently affected by congenital and adquired cardiopathies. The main objective of this study was the standardization and evaluation of the echocardiographic parameters in unidimensional $(M)$ mode, from clinically normal Miniature Poodle dogs. Thirthy Miniature Poodle dogs, 09 males and 21 females ageing between 2 and 7 years old $(3.87 \pm 1.55)$, and weight varying from 2.0 to 8.7 kilogram (4.49 \pm 1.38$)$ were studied. To be included in this study, physical exam, hemogram, biochemical profile, urinalysis, detection of circulating microfilaries as well as ELISA test for Dirofilaria immitis, electrocardiographic, radiographic exams and meansurement of the blood pressure were performed. After the analysis of obtained echocardiographic results, reference values for the echocardiographic data, in healthy Miniature Poodle dogs, were determined. The statistical analysis also showed that factors, as weight and height, may have some influence on the echocardiographic parameters.

Key words: echocardiography, Miniature Poodle, M mode, dogs.

\section{INTRODUÇÃO}

No Brasil, a população canina da raça Poodle, principalmente a variação miniatura, cresce em progressão geométrica, sendo isso facilmente observado na rotina do atendimento de hospitais veterinários como o da Faculdade de Medicina Veterinária e Zootecnia da Universidade de São Paulo (HOVET-FMVZ/USP) e nos estabelecimentos veterinários privados da cidade de São Paulo. Segundo LARSSON et al. (2000), dentre os cães atendidos no Serviço de Cardiologia do HOVET-FMVZ/USP, os Poodles, foram os mais comumente acometidos por cardiopatias.

\footnotetext{
${ }^{1}$ Centro Universitário das Faculdades Metropolitanas Unidas (UniFMU). Rua Itaipú, 354 casa 11, Mirandópolis, SP, Brasil. CEP: 04052-010. E-mail: rjyamato@uol.com.br. Autor para correspondência

${ }^{2}$ Departamento de Clínica Médica FMVZ-USP, São Paulo, SP, Brasil.

${ }^{3}$ Departamento de Cirurgia FMVZ-USP, São Paulo, SP, Brasil.

${ }^{4}$ Autônomo.
} 
Os animais da raça Poodle são freqüentemente acometidos por cardiopatias congênitas, como a persistência do ducto arterioso (GOODWIN, 1995; SISSON et al., 2000), a Tetralogia de Fallot (GOODWIN, 1995), e por cardiopatias adquiridas, como a endocardiose da valva mitral (KITTLESON, 1998; LARSSON et al., 2000). Ainda é possível observar nos cães Poodles, o aparecimento de doenças crônicas que apresentam repercussões importantes no aparelho cardiovascular, como as disfunções da adrenal, a insuficiência renal, o diabetes mellitus e as neoplasias (SMITH et al., 1995; KIENLE, 1998b).

Os primeiros estudos ecocardiográficos na espécie canina foram realizados em animais saudáveis com o objetivo de padronizar e correlacionar as medidas cardíacas em modo M e os índices da função cardíaca com a superfície e o peso corpóreo (BOON et al., 1983; LOMBARD, 1984). Porém, notou-se que, por influência das diversas conformações corpóreas existentes nos cães, a variabilidade das medidas ecocardiográficas estava presente não somente entre as espécies animais, mas também entre as raças caninas.

MORRISON et al. (1992) realizaram um estudo no qual foram avaliadas quatro raças caninas, o Pembroke Welsh Corgi, o Miniature Poodle, o Afghan Hound e o Golden Retriever, com o objetivo de estabelecer os valores de normalidade para as medidas ecocardiográficas, em modo M, e comparar as variações destas, entre as quatro raças estudadas. Os autores concluíram que a raça, considerando o peso corpóreo, é fator importante que influencia, de forma significativa, as mensurações dos parâmetros ecocardiográficos dos cães, com exceção ao diâmetro interno do ventrículo direito. Concluíram ainda que variações destas mensurações podem ocorrer mesmo em animais da mesma raça, devido à variação do tamanho e do peso corpóreo. Outros estudos foram realizados com o objetivo comum de padronizar os valores ecocardiográficos em modo $\mathrm{M}$ para algumas raças caninas, dentre elas, o Dobermann, o English Cocker Spaniel e o Beagle (CALVERT \& BROWN, 1986; GOODING et al., 1986; CRIPPA et al., 1992), além de avaliar as alterações ecocardiográficas que podem ser indicativas de cardiopatias ou apenas serem características inerentes à raça estudada (BONAGURA \& FUENTES, 2000; PAGE et al., 1993). Devido a diversas variáveis que podem influenciar de maneira significativa os parâmetros ecocardiográficos, entendese que haja necessidade de padronizar as medidas ecocardiográficas para cada raça da espécie canina.

Face ao significativo aumento populacional dos cães raça Poodle miniatura, às cardiopatias congênitas e adquiridas que os acomete, à variabilidade dos parâmetros ecocardiográficos entre as raças caninas e à escassez de trabalhos visando ao estudo ecocardiográfico ,na raça Poodle miniatura, o presente trabalho teve como escopo padronizar e estudar os parâmetros ecocardiográficos, em modo $\mathrm{M}$, dos cães da raça Poodle miniatura, clinicamente sadios.

\section{MATERIAL E MÉTODOS}

Foram incluídos, neste estudo, os resultados de 30 cães Poodle miniatura, com altura entre 25,0 a $38,0 \mathrm{~cm}$ (AMERICAN KENNEL CLUB, [s.d.]), clinicamente sadios, sendo 9 machos e 21 fêmeas, dos quais apenas uma fêmea era castrada, com idades entre 2 e 7 anos e peso corpóreo variando entre 2,0 a $8,7 \mathrm{~kg}$. Os animais foram selecionados a partir de canis de criadores da grande São Paulo e de cães que receberam alta dos diversos serviços do HOVET/FMVZ-USP.

O exame ecocardiográfico foi realizado em aparelho HITACHI ${ }^{\circledR}$ modelo EUB 515-A, equipamento compacto com recursos para estudo em modo $\mathrm{B}$, modo M e Doppler (pulsado, fluxo colorido e contínuo) e transdutor de alta densidade multi-freqüencial (3,5 e $5 \mathrm{Mhz})$.

Vídeo Graphic printer preto e branco $\left(\mathrm{SONY}^{\circledR}\right)$ e aparelho de vídeo cassete $\left(\mathrm{SHARP}^{\circledR}\right)$ foram utilizados para o registro das imagens ecocardiográficas, devidamente identificadas, para análise detalhada posteriormente aos exames.

Os animais selecionados foram previamente submetidos aos exames físico, radiográfico, eletrocardiográfico, mensuração da pressão arterial, exame de urina, dosagem sérica de uréia, creatinina, alanina aminotransferase, fosfatase alcalina, proteína total, albumina e bilirrubina, hemograma, pesquisa de microfilárias circulantes e de antígenos contra Dirofilaria immitis. Após essa avaliação, aqueles considerados hígidos foram submetidos ao exame ecocardiográfico, que foi realizado conforme recomendações da Echocardiography Committee of the Specialty of Cardiology - American College of Veterinary Internal Medicine (THOMAS et al., 1993), da American Society of Echocardiography (ASE) (BOON, 1998a) e autores consultados (BOON,1998b; BONAGURA, 1983; BONAGURA \& FUENTES, 2000).

As medidas ecocardiográficas em modo $\mathrm{M}$, realizadas no presente estudo, foram a freqüência cardíaca (FC), o diâmetro do ventrículo esquerdo na diástole (DVEd) e na sístole (DVEs), o septo interventricular na diástole (SIVd) e na sístole (SIVs), a parede livre do ventrículo esquerdo na diástole (PVEd) e na sístole (PVEs), o diâmetro da artéria aorta ou raiz da aorta (Ao), o diâmetro do átrio esquerdo (AE), a relação das medidas do AE e Ao (AE/Ao), a relação das medidas do SIVd e da PVEd (SIVd/PVEd), a relação 
das medidas do SIVs e da PVEs (SIVs/PVEs), a distância entre o ponto $\mathrm{E}$ do folheto anterior da valva mitral e o septo interventricular (dES) e o tempo de ejeção do ventrículo esquerdo (TEVE), sendo essas mensurações ilustradas na figura 1 .

Os índices da função cardíaca calculados, com os dados obtidos em modo $\mathrm{M}$, foram a velocidade de encurtamento circunferencial da fibra cardíaca (Vecf), a fração de encurtamento do ventrículo esquerdo (FE) e o engrossamento sistólico do septo interventricular (PSIV) e parede do ventrículo esquerdo (PPVE), conforme fórmulas demonstradas a seguir:

a) $\mathrm{FE} \%=[(\mathrm{DVEd}-\mathrm{DVEs}) / \mathrm{DVEd}] \times 100$

b) Vecf $=[($ DVEd - DVEs $) /($ TEVE $x$ DVEd $)]$

c) $\mathrm{PPVE} \%=[(\mathrm{PVEs}-\mathrm{PVEd}) / \mathrm{PVEd}] \times 100$

d) PSIV $=[($ SIVs - SIVd $) /$ SIVd $] \times 100$

Os valores dos parâmetros ecocardiográficos obtidos neste estudo foram submetidos à análise estatística descritiva, para o cálculo do valor médio e do desvio padrão. Calculouse, ainda, o coeficiente de correlação de Pearson (r), com a finalidade de avaliar a existência de correlação entre as variáveis peso corpóreo, altura e idade, e os parâmetros ecocardiográficos mensurados em modo M. Para o cálculo do r, estabeleceu-se o nível de significância igual a 0,05 (JEKEL et al., 1996).

\section{RESULTADOS E DISCUSSÃO}

Os valores das mensurações, assim como as médias obtidas dos parâmetros ecocardiográficos em modo $M$ de cães da raça Poodle miniatura, são apresentados na tabela 1 .

A medicina veterinária estuda as diversas espécies animais, que apresentam uma grande variação nas características morfológicas e fisiológicas, entre si, como também entre as diversas raças de cada espécie animal. Os cães, por sua vez, possuem uma grande variedade de raças e de tamanhos, abrangendo desde o pequeno Chihuahua até o gigante Bull Mastiff. Diante deste contexto, é lógico admitir-se que as medidas ecocardiográficas também variam de acordo com a espécie e o tamanho do animal. Outros fatores também podem influenciar as medidas cardíacas, como a idade, o peso, a superfície e a conformação corpórea (BOON et al., 1983; MORRISON et al., 1992; KIENLE, 1998a).

Dentre as raças caninas, o Poodle miniatura, sem dúvida, tornou-se muito popular nas grandes capitais do Brasil, a exemplo da cidade de São Paulo. Este aumento populacional é facilmente observado na rotina das clínicas veterinárias particulares e instituições de ensino como a FMVZ-USP, pois segundo LARSSON et al. (2000), o Poodle foi a principal raça acometida pela endocardiose valvar mitral, e a mais freqüentemente atendida pelo Serviço de Cardiologia do HOVET/FMVZ-USP, no período de agosto de 1998 a janeiro de 2000. Deve-se ressaltar que variações nas mensurações ecocardiográficas podem ocorrer entre os animais da mesma espécie e raças, mesmo considerando a variação em consequiência do peso corpóreo (MORRISON et al., 1992; BONAGURA \& FUENTES, 2000).

Os resultados obtidos neste estudo são muito similares àqueles referidos por MORRISON et al. (1992). As médias dos parâmetros ecocardiográficos em modo M, obtidas no presente estudo, não foram comparadas estatisticamente com o estudo realizado por MORRISON et al. (1992), pois se entende que o estudo estatístico não é válido nesta ocasião, tendo em vista que variações referentes à metodologia e ao equipamento podem influenciar de maneira significativa nestes resultados. Porém, realizou-se apenas uma comparação entre os resultados obtidos neste estudo e os obtidos por MORRISON et al. (1992), conforme demonstrados na tabela 2. Esta observação sugere que a variabilidade entre examinadores pode existir, mas parece não influenciar de maneira significativa nas medidas ecocardiográficas (O’GRADY et al., 1986).

O sexo dos cães não foi pré-requisito para a inclusão neste estudo, pois estudos prévios sugerem que esta variável não exerce influência sobre as medidas ecocardiográficas (LOMBARD, 1984; MORRISON et al., 1992). Não se incluiu a mensuração da dimensão interna do ventrículo direito (VD) pois a morfologia desta câmara cardíaca não é dotada do mesmo padrão de simetria como a do ventrículo esquerdo (VE), fato este que pode originar grande variação na mensuração deste parâmetro ecocardiográfico.

Realizou-se o cálculo do coeficiente de correlação de Pearson (r) (JEKEL et al., 1996) para os parâmetros anteriormente citados, com a finalidade de estudar a existência de correlação linear das variáveis peso, altura e idade sobre os parâmetros ecocardiográficos em modo M. Este coeficiente pode variar seu valor entre $+1 \mathrm{e}-1$, sendo o valor +1 indicativo de uma correlação positiva perfeita ou proporcional e o valor -1 uma correlação negativa perfeita ou inversamente proporcional. Considerou-se correlação positiva e significativa, os valores de " $r$ " maiores ou iguais a 0,40 e "P" menores ou iguais a 0,05. Após a análise dos dados obtidos, sugere-se que o peso possui correlação linear positiva e significativa $(0,49 \leq \mathrm{r} \leq 0,83$ e $0,0001 \leq \mathrm{P} \leq 0,0063)$ em relação ao diâmetro do ventrículo esquerdo na diástole (DVEd) e na sístole (DVEs), à espessura do septo interventricular na diástole (SIVd) e na sístole (SIVs), à espessura da 


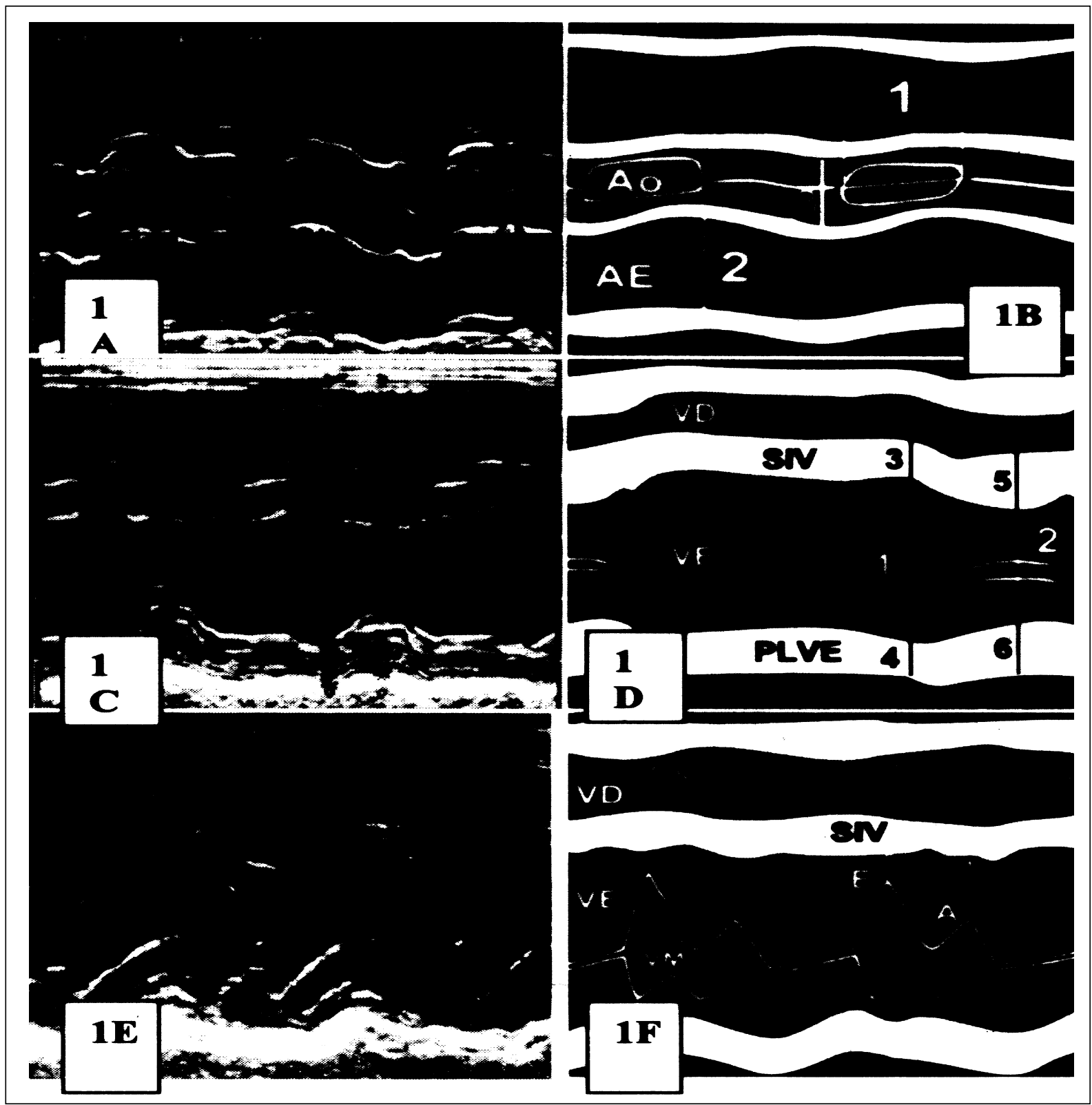

Figura 1 - 1A e 1B: Foto e desenho esquemático das mensurações realizadas em modo $\mathrm{M}$ da aorta e átrio esquerdo e foto do ecocardiograma de corte longitudinal para-esternal esquerdo da via de saída do ventrículo esquerdo, onde $1=$ tempo de ejeção do ventrículo esquerdo, 2 = diâmetro do átrio esquerdo, 3 = diâmetro da artéria ou raiz da aorta, $\mathrm{AE}=$ átrio esquerdo e Ao = artéria aorta. 1C e 1D:Foto e desenho esquemático das mensurações realizadas em modo $\mathrm{M}$ do ventrículo esquerdo e foto do ecocardiograma do corte transversal para-esternal direito do ventrículo esquerdo, onde 1 = diâmetro da cavidade do ventrículo esquerdo em diástole, 2 = diâmetro da cavidade do ventrículo esquerdo em sístole, 3 = espessura do septo interventricular em diástole, 4 = espessura da parede do ventrículo esquerdo em diástole, $5=$ espessura do septo interventricular em sístole, $6=$ espessura da parede do ventrículo esquerdo em sístole, $\mathrm{VE}=$ ventrículo esquerdo, $\mathrm{VD}=$ ventrículo direito, $\mathrm{SIV}=$ septo interventricular e PVE = parede do ventrículo esquerdo. 1E e 1F: Foto e desenho esquemático das mensurações realizadas em modo $\mathrm{M}$ da válvula mitral, onde 1 = distância entre o ponto $\mathrm{E}$ do folheto anterior da valva mitral e o septo interventricular, $\mathrm{VD}=$ ventrículo direito, $\mathrm{VE}=$ ventrículo esquerdo, $\mathrm{SIV}=$ septo interventricular, $\mathrm{VM}=$ válvula mitral e $\mathrm{A}=$ ponto A do folheto anterior da valva mitral.

Fonte: YAMATO, R.J. Serviço de Cardiologia - Departamento de Clínica Médica - FMVZ/USP, 2001

Fonte - Modificado de BOON, J.A. 1998b

Ciência Rural, v.36, n.1, jan-fev, 2006. 
Tabela 1 - Mensurações, índices da função cardíaca, proporções entre os parâmetros ecocardiográficos, médias e desvio padrão (DP) dos parâmetros ecocardiográficos em modo M, de 30 cães sadios da raça Poodle miniatura - São Paulo - nov.2000 - out. 2001

\begin{tabular}{|c|c|c|c|c|c|c|c|c|c|c|c|c|c|c|c|c|c|c|}
\hline NA & $\begin{array}{l}\text { FC } \\
\text { (bpm) }\end{array}$ & $\begin{array}{l}{ }^{1} \mathrm{DVEd}^{2} \\
(\mathrm{~cm})\end{array}$ & $\begin{array}{l}\mathrm{DVEs}^{3} \\
(\mathrm{~cm})\end{array}$ & $\begin{array}{l}\text { SIVd } \\
(\mathrm{cm})\end{array}$ & $\begin{array}{c}{ }^{4} \mathrm{SIV} s \\
(\mathrm{~cm})\end{array}$ & $\begin{array}{l}{ }^{5} \text { PVEd } \\
(\mathrm{cm})\end{array}$ & $\begin{array}{c}{ }^{6} \text { PVEs } \\
(\mathrm{cm})\end{array}$ & $\begin{array}{l}{ }^{7} \text { Ao } \\
(\mathrm{cm})\end{array}$ & $\begin{array}{l}{ }^{8} \mathrm{AE} \\
(\mathrm{cm})\end{array}$ & ${ }^{9} \mathrm{AE} / \mathrm{A}^{1}$ & $\begin{array}{l}{ }^{10} \text { SIVd/ } \\
\text { PVEd }^{11}\end{array}$ & $\begin{array}{l}\text { SIVs/ } \\
\text { PVEs }\end{array}$ & $\begin{array}{r}\mathrm{dES} \\
{ }^{2}(\mathrm{~cm})\end{array}$ & $\begin{array}{c}{ }^{13} \mathrm{TEVE} \\
(\mathrm{s})\end{array}$ & ${ }^{14} \mathrm{FE}^{15}(\%)$ & $\begin{array}{l}\text { Vecf } \\
(\mathrm{cm} / \mathrm{s})\end{array}$ & $\begin{array}{c}{ }^{16} \mathrm{PPVE} \\
(\%)\end{array}$ & $\begin{array}{c}{ }^{17} \mathrm{PSIV}^{18} \\
(\%)\end{array}$ \\
\hline$\overline{01}$ & 114 & ,45 & 1,50 & 0,55 & 1,01 & 0,56 & 0,86 & 1,46 & 1,42 & 0,97 & 0,98 & 1,17 & 0,37 & 0,28 & 38,90 & 1,38 & 53,57 & 83,64 \\
\hline 02 & 66 & 1,98 & 1,19 & 0,49 & 0,70 & 0,60 & 1,06 & 1,34 & 1,53 & 1,14 & 0,82 & 0,66 & 0,30 & 0,22 & 39,90 & 1,81 & 76,67 & 42,86 \\
\hline 03 & 100 & 2,30 & 1,55 & 0,56 & 0,78 & 0,57 & 0,91 & 1,32 & 1,34 & 1,02 & 0,98 & 0,86 & 0,32 & 0,19 & 36,75 & 1,72 & 59,65 & 39,29 \\
\hline 04 & 150 & 1,96 & 1,18 & 0,52 & 0,79 & 0,53 & 0,89 & 1,32 & 1,32 & 1,00 & 0,98 & 0,89 & 0,32 & 0,45 & 39,60 & 0,88 & 67,92 & 51,92 \\
\hline 05 & 90 & 2,50 & 1,56 & 0,63 & 0,88 & 0,57 & 0,96 & 1,49 & 1,78 & 1,19 & 1,11 & 0,92 & 0,43 & 0,19 & 37,46 & 1,98 & 68,42 & 39,68 \\
\hline 06 & 100 & 1,87 & 1,00 & 0,43 & 0,74 & 0,43 & 0,73 & 1,06 & 1,18 & 1,11 & 1,00 & 1,01 & 0,23 & 0,21 & 46,37 & 2,22 & 69,77 & 72,09 \\
\hline 07 & 95 & 2,26 & 1,30 & 0,44 & 0,76 & 0,45 & 0,70 & 1,19 & 1,21 & 1,02 & 0,98 & 1,09 & 0,23 & 0,25 & 42,55 & 1,70 & 55,56 & 72,73 \\
\hline 08 & 99 & 2,39 & 1,48 & 0,63 & 0,93 & 0,57 & 0,91 & 1,47 & 1,47 & 1,00 & 1,11 & 1,02 & 0,32 & 0,21 & 37,85 & 1,81 & 59,65 & 47,62 \\
\hline 09 & 102 & 2,54 & 1,55 & 0,52 & 0,93 & 0,52 & 0,83 & 1,45 & 1,47 & 1,01 & 1,00 & 1,12 & 0,33 & 0,31 & 38,87 & 1,26 & 59,62 & 78,85 \\
\hline 10 & 93 & 2,03 & 1,23 & 0,44 & 0,73 & 0,46 & 0,70 & 1,25 & 1,37 & 1,10 & 0,96 & 1,04 & 0,26 & 0,32 & 39 & 1,23 & 52,17 & 65,91 \\
\hline 11 & 159 & 2,50 & 1,36 & 0,65 & 1,02 & 0,66 & 1,06 & 1,40 & 1,42 & 1,01 & 0,98 & 0,96 & 0,32 & 0,24 & 45 & 10 & 60,61 & 56,92 \\
\hline 12 & 121 & 2,28 & 1,38 & 0,49 & 0,86 & 0,54 & 0,79 & 1,45 & 1,49 & 1,03 & 0,91 & 1,09 & 0,00 & 0,21 & & 8 & 46,30 & 75,51 \\
\hline 13 & 104 & 3,12 & 1,76 & 0,62 & 1,13 & 0,65 & 1,11 & 1,51 & 1,53 & 1,01 & 0,95 & 1,02 & 0,39 & 0,26 & & 8 & 70,77 & 82,26 \\
\hline 14 & 143 & 1,87 & 1,17 & 0,45 & 0,74 & 0,43 & 0,74 & 1,13 & 1,49 & 1,32 & 1,05 & 1,00 & 0,23 & 0,19 & 3 & 7 & 72,09 & 64,44 \\
\hline 15 & 86 & 2,23 & 1,25 & 0,48 & 0,74 & 0,47 & 0,84 & 1,25 & 1,36 & 1,09 & 1,02 & 0,88 & 0,19 & 0,22 & 0 & 10 & 78,72 & 54,17 \\
\hline 16 & 86 & 2,21 & 1,25 & 0,43 & 0,81 & 0,43 & 0,62 & 1,06 & 1,38 & 1,30 & 1,00 & 1,31 & 0,19 & 0,24 & 43, & 1 & 19 & 88,37 \\
\hline 17 & 128 & 2,38 & 1,39 & 0,48 & 0,83 & 8 & 0,83 & 1,36 & 1,45 & 1,07 & 1 & 1,00 & 17 & 0,24 & 41,5 & 3 & 92 & 92 \\
\hline 18 & 124 & 1,93 & 0,91 & 0,47 & 0,89 & 0,49 & 0,83 & 1,08 & 1 , & 1,10 & 0,96 & 1,07 & 0,09 & 0,24 & 52 & 2,20 & 69,39 & 89,36 \\
\hline 19 & 6 & 2,51 & 1,75 & 0,48 & 0,72 & 0,50 & 0,79 & 1,32 & 1,47 & 1,11 & 0,96 & 0,91 & 0,23 & 0,21 & 30,15 & 1,44 & 58,00 & 50,00 \\
\hline 20 & 105 & 2,44 & 1,40 & 0,47 & 0,91 & 0,47 & 0,81 & 1,28 & 1,38 & 1,08 & 1,00 & 1,12 & 0,23 & 0,24 & 42,62 & 1,78 & 72,34 & 93,62 \\
\hline 21 & 107 & 2,44 & 1,42 & 0,55 & 0,83 & 0,55 & 0,85 & 1,28 & 1,45 & 1,13 & 1,00 & 0,98 & 0,23 & 0,22 & 41,80 & 1,90 & 54,55 & 50,91 \\
\hline 22 & 72 & 1,99 & 1,21 & 0,55 & 0,68 & 0,50 & 0,82 & 1,19 & 1,38 & 1,16 & 1,10 & 0,83 & 0,28 & 0,25 & 39,13 & 1,57 & 64,00 & 23,64 \\
\hline 23 & 95 & 2,40 & 1,29 & 0,47 & 0,83 & 0,46 & 0,84 & 1,30 & 1,59 & 1,22 & 1,02 & 0,99 & 0,26 & 0,30 & 46,20 & 1,54 & 82,61 & 76,60 \\
\hline 24 & 94 & 2,64 & 1,56 & 0,51 & 0,80 & 0,48 & 0,87 & 1,45 & 1,51 & 1,04 & 1,0 & 0,92 & 0,17 & 0,17 & 40,90 & 2,41 & 81,25 & 56,86 \\
\hline 25 & 112 & 2,40 & 1,40 & 0,57 & 0,96 & 0,55 & 0,85 & 1,25 & 1,28 & 1,02 & 1,04 & 1,13 & 0,23 & 0,28 & 41,67 & 1,49 & 54,55 & 68,42 \\
\hline 26 & 110 & 2,31 & 1,44 & 0,52 & 0,90 & 0,50 & 0,77 & 1,28 & 1,3 & 1,06 & 1,0 & 1,17 & 0,15 & 0,24 & 37,65 & 1,57 & 54,00 & 73,08 \\
\hline 27 & 87 & 2,53 & 1,57 & 0,55 & 0,88 & 0,48 & 0,86 & 1,51 & 1,53 & 1,01 & 1,15 & 1,02 & 0,21 & 0,32 & 44,10 & 1,19 & 79,17 & 60,00 \\
\hline 28 & 97 & 2,18 & 1,27 & 0,50 & 0,89 & .53 & 0,83 & 1,38 & 1,40 & 1,01 & 0,94 & 1,07 & 0,20 & 0,20 & 41,50 & 2,09 & 56,60 & 78,00 \\
\hline 29 & 103 & 2,40 & 1,42 & 0,51 & 0,96 & 0,55 & 0,81 & 1,36 & 1,36 & 1,00 & 0,93 & 1,19 & 0,26 & 0,18 & 40,83 & 2,27 & 47,27 & 88,24 \\
\hline 30 & 103 & 2,40 & 1,33 & 0,52 & 0,82 & 0,53 & 0,80 & 1,35 & 1,35 & 1,00 & 0,98 & 1,03 & 0,28 & 0,21 & 44,63 & 2,12 & 50,94 & 57,69 \\
\hline M & 104 & 2,31 & 1,37 & 0,52 & 0,85 & 0,52 & 0,84 & 1,32 & 1,42 & 1,08 & 1,00 & 1,02 & 0,25 & 0,24 & 41,20 & 1,75 & 63,11 & 65,19 \\
\hline DP & 22 & 0,27 & 0,19 & 0,06 & 0,11 & 0,06 & 0,11 & 0,13 & 0,12 & 0,09 & 0,07 & 0,13 & 0,09 & 0,06 & 4,02 & 0,35 & 10,98 & 17,35 \\
\hline
\end{tabular}

Legenda: $\mathrm{NA}=$ número do animal; $\mathrm{bpm}=$ batimentos por minuto; $\mathrm{cm}=$ centímetros; $\mathrm{s}=$ segundos; ${ }^{1}=$ freqüência cardíaca; ${ }^{2}=$ diâmetro do ventrículo esquerdo na diástole; ${ }^{3}=$ diâmetro do ventrículo esquerdo na sístole; ${ }^{4}=$ espessura do septo interventricular na diástole; ${ }^{5}=$ espessura do septo interventricular na sístole $;{ }^{6}=$ espessura da parede do ventrículo esquerdo na diástole; ${ }^{7}=$ espessura da parede do ventrículo esquerdo na sístole $;{ }^{8}=$ diâmetro da artéria aorta; ${ }^{9}=$ diâmetro do átrio esquerdo $;{ }^{10}=$ relação das medidas de $\mathrm{AE}$ e Ao; ${ }^{11}=$ relação das medidas do SIVd e PVEd; ${ }^{12}=$ relação das medidas do SIVs e PVEs; ${ }^{13}$ = distância entre o ponto E do folheto anterior da valva mitral e o septo interventricular; ${ }^{14}=$ tempo de ejeção do ventrículo esquerdo; ${ }^{15}=$ fração de encurtamento do ventrículo esquerdo; ${ }^{16}=$ velocidade de encurtamento circunferencial da fibra cardíaca; ${ }^{17}=$ engrossamento sistólico da PVE; ${ }^{18}=$ engrossamento sistólico do SIV.

parede livre do ventrículo esquerdo na diástole (PVEd) e na sístole (PVEs), ao diâmetro da artéria aorta (Ao), ao diâmetro do átrio esquerdo (AE) e à distância entre o ponto $\mathrm{E}$ do folheto anterior da valva mitral e o septo interventricular (dES). Porém a relação AE/Ao, SIVd/ PVEd e SIVs/PVEs, o peso corpóreo revelou coeficientes de correlação negativos e não significativos $(-0,35 \leq \mathrm{r}$ $\leq 0,18$ e $0,33 \leq \mathrm{P} \leq 0,56$ ).

A altura também apresentou coeficientes de correlação positivos e significativos $(0,47 \leq \mathrm{r} \leq 0,72 \mathrm{e}$ $0,0001 \leq \mathrm{P} \leq 0,0081)$ em relação ao DVE na diástole e na sístole, à PVE na diástole e na sístole, à SIV na diástole e na sístole, à Ao e à dES, enquanto que, em relação ao $\mathrm{AE}$, à $\mathrm{AE} / \mathrm{Ao}$, à SIVd/PVEd, à SIVs/PVEs, a altura mostrou coeficientes negativos ou não significativos $(-0,46 \leq \mathrm{r} \leq 0,25$ e $0,011 \leq \mathrm{p} \leq 0,29)$.

A idade do animal, por sua vez, após o cálculo dos coeficientes de Pearson, não demonstrou correlação significativa $(-0,0007 \leq \mathrm{r} \leq 0,33$ e $0,07 \leq \mathrm{P} \leq$
0,99) com o SIVd, o SIVs, a PVEd, a PVEs, o AE, Ao, a AE/Ao e a dES; enquanto foi observada uma correlação positiva $(0,40 \leq \mathrm{r} \leq 0,51$ e $0,0041 \leq \mathrm{P} \leq 0,03)$ em relação à DVEd e ao DVEs.

A ausência da correlação entre a idade e a maioria dos parâmetros ecocardiográficos era esperada, pois a faixa etária entre dois e sete anos foi determinada com a finalidade de se minimizar ao máximo a influência de filhotes e animais idosos. Os índices da função do VE, quais sejam FE, TEVE, Vecf, PPVE e a PSIV, não apresentaram correlação positiva significativa $(r=-0,36$ a $0,23 / \mathrm{P}=0,047$ a 0,22 ) com as variáveis peso, altura e idade. Realizou-se, também, a análise de correlação entre a freqüência cardíaca e a FE, na qual não se observou correlação entre os dois parâmetros $(r=0,79 / \mathrm{p}=0,18)$, análise esta realizada devido à idéia intuitiva de que quanto maior a FC maior a FE. Esta idéia não é verdadeira devido ao fato de que a FE representa a porcentagem de redução do diâmetro do ventrículo esquerdo na 
Tabela 2 - Comparação dos parâmetros ecocardiográficos em modo M, obtidos nesta pesquisa e MORRISON et al., 1992, de cães da raça Poodle miniatura - São Paulo - nov.2001

\begin{tabular}{lcc}
\hline & Parâmetros ecocardiográficos em modo M de cães da raça Poodle miniatura \\
\hline Parâmetros & $\begin{array}{c}\text { Valores obtidos nesta pesquisa valor médio(valor } \\
\text { mínimo - valor máximo) }\end{array}$ & $\begin{array}{c}\text { MORR et al, 1992 valor médio(valor } \\
\text { mínimo - valor máximo) }\end{array}$ \\
FC $^{1}(\mathrm{bpm})$ & $104(65-159)$ & $150(100-200)$ \\
$\operatorname{DVEd}^{2}(\mathrm{~cm})$ & $2,31(1,87-3,12)$ & $2,00(1,60-2,80)$ \\
$\operatorname{DVEs}^{3}(\mathrm{~cm})$ & $1,37(0,91-1.76)$ & $1,00(0,80-1,60)$ \\
$\operatorname{SIVd}^{4}(\mathrm{~cm})$ & $0,52(0,43-0,65)$ & $0,50(0,40-0,60)$ \\
$\operatorname{SIVs}^{5}(\mathrm{~cm})$ & $0,85(0,68-1,13)$ & $0,80(0,60-1,00)$ \\
$\operatorname{PVEd}^{6}(\mathrm{~cm})$ & $0,52(0,43-0,66)$ & $0,50(0,40-0,60)$ \\
$\operatorname{PVEs}^{7}(\mathrm{~cm})$ & $0,84(0,62-1,11)$ & $0,80(0,60-1,00)$ \\
$\operatorname{Ao}^{8}(\mathrm{~cm})$ & $1,32(1,06$ a 1,51$)$ & $1,00(0,80-1,30)$ \\
$\operatorname{AE}^{9}(\mathrm{~cm})$ & $1,42(1,18$ a 1,78$)$ & $1,20(0,80-1,80)$ \\
dES $^{10}(\mathrm{~cm})$ & $0,25(0,0$ a 0,43$)$ & $0(0-0,20)$ \\
FE $^{11}(\%)$ & $41,20(30,15$ a 52,85$)$ & $47,0(35,0-57,0)$ \\
\hline
\end{tabular}

Legenda: bpm $=$ batimentos por minuto $; \mathrm{cm}=$ centímetros $;{ }^{1}=$ freqüência cardíaca $;{ }^{2}=$ diâmetro do ventrículo esquerdo na diástole ${ }^{3}=$ diâmetro do ventrículo esquerdo na sístole; ${ }^{4}=$ espessura do septo interventricular na diástole; ${ }^{5}=$ espessura do septo interventricular na sístole; ${ }^{6}=$ espessura da parede do ventrículo esquerdo na diástole $;{ }^{7}=$ espessura da parede do ventrículo esquerdo na sístole; ${ }^{8}=$ diâmetro da artéria aorta; ${ }^{9}=$ diâmetro do átrio esquerdo; ${ }^{10}=$ distância entre o ponto $\mathrm{E}$ do folheto anterior da valva mitral e o septo interventricular; ${ }^{11}=$ fração de encurtamento do ventrículo esquerdo.

sístole, e não a mensuração da contratilidade miocárdica como um todo (BOON, 1998a). Sugere-se que mais estudos sejam realizados com a finalidade de se verificar, isoladamente ou conjuntamente, a influência das variáveis peso, altura, idade e a frequiência cardíaca sobre os parâmetros ecocardiográficos, para os animais da espécie e de diversas raças caninas.

Os resultados obtidos neste estudo, relativos à influência do peso corpóreo e os parâmetros ecocardiográficos, em modo M, e os índices da função do VE, corroboram com os observados por BOON et al. (1983), LOMBARD (1984) e MORRISON etal. (1992). Esses resultados são esperados, uma vez que as dimensões cardíacas devem ser proporcionais ao tamanho do animal e seus índices de funcionalidade seguem um padrão para cada espécie animal.

\section{CONCLUSÕES}

Na análise dos resultados obtidos e por meio da metodologia utilizada no presente estudo, foi possível concluir que os padrões ecocardiográficos em modo M para cães Poodle miniatura, sadios, adultos e com peso corpóreo médio 4,49 1,38kg, são: $\mathrm{FC}=104$ $\pm 22 \mathrm{bpm}, \mathrm{DVEd}=2,31 \pm 0,27 \mathrm{~cm}, \mathrm{DVEs}=1,37 \pm 0,19 \mathrm{~cm}$, $\mathrm{SIVd}=0,52 \pm 0,06 \mathrm{~cm}$, SIVs $=0,85 \pm 0,11 \mathrm{~cm}, \mathrm{PVEd}=0,52$ $\pm 0,06 \mathrm{~cm}, \mathrm{PVEs}=0,84 \pm 0,11 \mathrm{~cm}, \mathrm{Ao}=1,32 \pm 0,13 \mathrm{~cm}, \mathrm{AE}$ $=1,42 \pm 0,12 \mathrm{~cm}, \mathrm{AE} / \mathrm{Ao}=1,08 \pm 0,09, \mathrm{SIVd} / \mathrm{PVEd}=1,00$ $\pm 0,07$, SIVs $/ S I V s=1,02 \pm 0,13 \mathrm{~cm}, \mathrm{dES}=0,25 \pm 0,09 \mathrm{~cm}$, $\mathrm{TEVE}=0,24 \pm 0,06 \mathrm{~s}, \mathrm{FE}=41,20 \pm 4,02 \%$, Vecf $=1,75 \pm$ $0,35 \mathrm{~cm} / \mathrm{s}, \mathrm{PSIV}=65,19 \pm 17,35 \%, \mathrm{PPVE}=63,11 \pm 10,98$; as variáveis peso corpóreo e altura sugerem ter influência positiva sobre os parâmetros ecocardiográficos, em modo $\mathrm{M}$, dos cães da raça Poodle miniatura, exceção feita aos índices da função do VE, sendo a influência do peso corpóreo mais evidente que a da altura; a fração de encurtamento, índice de função do ventrículo esquerdo, independe da frequiência cardíaca; parâmetros ecocardiográficos, mensurados em modo $M$, são passíveis de variação dentro de uma mesma raça canina.

\section{AGRADECIMENTOS E APRESENTAÇÃO}

Estudo realizado na Faculdade de Medicina Veterinária e Zootecnia da Universidade de São Paulo (FMVZ-USP) com apoio financeiro da Fundação de Amparo à Pesquisa do Estado de São Paulo- FAPESP (processo n ${ }^{\circ}$ 99/02666-7).

\section{REFERÊNCIAS}

AMERICAN KENNEl CLUB. Poodle. New York, [s.d.]. Capturado em 15 ago. 2001. Online. Disponível na Internet http://www.akc.org.

BONAGURA, J.D. M-Mode echocardiography. Veterinary Clinics of North America. Small Animal Practice, v.13, n.2, p.299-319, 1983.

BONAGURA, J.D.; FUENTES, V.L. Echocardiography. In: ETTINGER, S.J.; FELDEMAN, E.C. Textbook of veterinary internal medicine. 5.ed. Philadelphia: Saunders, 2000. V.1, p. 834-873.

BOON, J.A. Evaluation of size, function, and hemodynamics. In: _-___. Manual of veterinary echocardiography. Baltimore: Williams and Wilkins, 1998a. p.151-260. 
BOON, J.A. The echocardiographic examination. In: Manual of veterinary echocardiography. Baltimore: Williams and Wilkins, 1998b. p.35-150.

BOON, J.A. et al. Echocardiography indices in the normal dog. Veterinary Radiology, v.24, n.5, p.214-221, 1983.

CALVERT, C.A.; BROWN, J. Use of M-mode echocardiography in the diagnosis of congestive cardiomyopathy in Doberman Pinschers. Journal of American Veterinary Medical Association, v.189, n.3, p.293-297, 1986.

CRIPPA, L. et al. Echocardiographic parameters and indices in the normal Beagle dog. Laboratory Animals, v.26, n.3, p.190$195,1992$.

GOODWIN, J.K. Congenital heart disease. In: MILLER, M.S.; TILLEY, L.P. Manual of canine and feline cardiology. 2.ed. Philadelphia: Saunders, 1995. p.271-294.

GOODING, J.P. et al. Echocardiography assessment of left ventricular dimensions in clinically normal English Cocker Spaniels. American Journal Veterinary Research, v.47, n.2, p.296-300, 1986.

JEKEL, F.J. et al. Análise bivariada. In: JEKEL, F.J. et al. Epidemiologia, bioestatística e medicina preventiva. Porto Alegre: Artes Médica Sul, 1996. p.155-177.

KIENLE, R.D. Echocardiography. In: KITTLESON, M.D.; KIENLE, R.D. Small animal cardiovascular medicine. St. Louis: Mosby, 1998a. p.95-117.

KIENLE, R.D. The effects of systemic disease on the cardiovascular system. In: KITTLESON, M.D.; KIENLE, R.D. Small animal cardiovascular medicine. St. Louis: Mosby, 1998b. p.552-560.

KITTLESON, M.D. Myxomatous atrioventricular valvular degeneration. In: KITTLESON, M.D.; KIENLE, R.D. Small animal cardiovascular medicine. St. Louis: Mosby, 1998. p.297-318.

LARSSON, M.H.M.A. et al. Estudo ecocardiográfico das cardiopatias mais freqüentemente diagnosticadas em espécimes caninas. Revista Brasileira de Ciência Veterinária, v.7, p.68, 2000. uplemento. (Apresentado no CONGRESSO BRASILEIRO DE CLÍNICOS VETERINÁRIOS DE PEQUENOS ANIMAIS, 21, 2000, Rio de Janeiro).

LOMBARD, C.W. Normal values of the canine M-mode echocardiogram. American Journal of Veterinary Research, v.45, n.10, p.2015-2018, 1984.

MORRISON, S.A. et al. Effect of breed and body weight on echocardiography values in four breeds of dogs of differing somatotype. Journal of Veterinary Internal Medicine, v. 6, n. 4, p. 220-224, 1992.

O'GRADY, M. R. et al. Quantitative cross-sectional echocardiography in the normal dog. Veterinary Radiology, v. 27 , n. 2 , p. $34-49,1986$.

PAGE, A. et al. Echocardiography values in the Greyhound. Australian Veterinary Journal, v. 70, n. 10, p. 361-364, 1993.

SISSON, D. D. et al. Congenital heart disease. In: ETTINGER, S. J.; FELDEMAN, E. C. Textbook of veterinary internal medicine. 5. ed. Philadelphia: Saunders, 2000. v. 1, p. 737787.

SMITH Jr., F. W. K. et al. Cardiovascular disorders in systemic disease. In: MILLER, M. S.; TILLEY, L. P. Manual of canine and feline cardiology. 2. ed. Philadelphia: Saunders, 1995. p. 295-342.

THOMAS, W.P. et al. Recommendations for standards in transthoracic two-dimensional echocardiography in the dog and cat. Journal of Veterinary Internal Medicine, v.7, n.4, p.247-252, 1993. 